\title{
Habilidades sociales en el espectro autista
}

\author{
Cesia BEJARANO CAJACHAGUA*
}

E.A.P. Psicología, Facultad de Ciencias de la Salud, Universidad Peruana Unión, Alt. Km 19; Carretera Central, Lurigancho, Lima

\begin{abstract}
RESUMEN
Este trabajo es de naturaleza teórica. El propósito fue identificar las características de las habilidades sociales con las que cuenta una persona del espectro autista y hasta qué punto éstas pueden desarrollarse en el mismo. Se revisan los aportes de investigadores como Reviere (1997), Meléndez (2009) y el DSM-IV (2002), quienes consideran al autismo como un trastorno generalizado de desarrollo de tipo neurológico que afecta tres funciones específicas relacionadas entre sí, las cuales se desarrollan durante los tres primeros años de su vida, éstas son: la interacción social, dificultad del lenguaje comunicativo y patrones de comportamiento, intereses y actividades restringidas repetitivas y estereotipadas. Asimismo, para la comprensión de las habilidades sociales las teorías de aprendizaje social y vicario, las cuales indican que el individuo posee una capacidad para percibir, comprender, descifrar y, posteriormente, responder a determinados estímulos sociales en general. Se concluye que ninguna persona está restringida a desarrollar estas habilidades que en definitiva son vitales para nuestra adaptación en el contexto que sea, de la misma forma una persona perteneciente al espectro autista puede poseer y desarrollar tan igual que otra persona "normal" las habilidades sociales.
\end{abstract}

Palabras clave: Efectos, televisión, niños.

\begin{abstract}
This work is theoretical in nature. The purpose was to identify the characteristics of the social skills that a person has autism spectrum and how these can be developed in it. We review the contributions of researchers as Reviere (1997), Melendez (2009) and the DSM-IV (2002), who think of autism as a pervasive developmental disorder of neurological affects three interrelated specific functions, which are develop during the first 3 years of life, these are: social interaction, communication and language difficulty patterns of behavior, interests and activities restricted repetitive and stereotyped. And for understanding social skills social learning theories and vicar, which indicate that the individual has the capacity to perceive, understand, decipher, and then respond to certain social stimuli in general. We conclude that no one is restricted to develop these skills that really are vital to our adaptation in the context that is, in the same way a person belonging to the autistic spectrum may possess and develop as well as other “normal” social skills .
\end{abstract}

Keywords: Effects, television, children.

\footnotetext{
* Correspondencia: cesy_11oct@hotmail.com
} 


\section{INTRODUCCIÓN}

En el mundo actual, los casos de autismo y otros trastornos relacionados son cada vez mayores. A medida que el tiempo pasa la tasa de afectados asciende. Se considera la cifra de 2 a 3 de cada mil personas que sufren del llamado espectro autista (Passerino, 2007). Algunas de las características principales del Trastorno del Espectro Autista (TEA) es la casi imposibilidad para crear vínculos sociales efectivos, ya sea en un ambiente familiar o escolar, en suma en un ambiente interpersonal. Así, su desarrollo integral se ve alterado según sea el nivel de gravedad. Sin embargo, la capacidad de socialización se encuentra privada más no ausente, quiere decir que estas personas al igual que otras son tan capaces de desarrollar habilidades sociales si solo si se le presta y da el entrenamiento correspondiente. Se comprende a las habilidades sociales como un conjunto de capacidades de actuación aprendida que se desarrolla en un contexto interpersonal, donde el aprendizaje se da por imitación o aprendizaje vicario. En este marco estudiaremos al trastorno del espectro autista y el desarrollo de las habilidades sociales, que según DSM-IV es un conjunto de alteraciones generalizadas basadas en el desarrollo integral del sujeto, las más marcadas y fundamentales son: alteraciones en la interacción social, alteraciones en la comunicación y patrones de comportamiento, intereses y actividades restringidos, repetitivos y estereotipados.

El objetivo de este trabajo es presentar las características básicas que engloban el espectro autista, los roles importantes que juegan las funciones cognitivas, de desarrollo motor, lenguaje, comunicativas, entre otras, en la instalación de habilidades sociales.

\section{Trastorno del Espectro Autista}

\section{Historia.}

En los años 1991, el suizo Eugen Bleuer utilizó por primera vez la palabra autismo para referirse a un proceso de separación de la realidad, que era substituida por alucinaciones. En su obra titulada "La demencia precoz o el grupo de las esquizofrenias”, presentaba al autismo como una de las manifestaciones posibles en casos de esquizofrenia (Ramos, 2010).

Asimismo, en el año 1943 Leo Kanner psiquiatra austriaco, publicó un trabajo referente al autismo, donde hace una diferenciación significativa entre autismo y esquizofrenia, asumiendo que el espectro autista constituye una entidad clínica independiente. Ahora bien, la publicación elaborada se basó en "Trastornos autistas del contacto afectivo”, en este apartado Kanner menciona que las causas desencadenantes resulta de los vínculos afectivos, desde este punto de vista, Kanner afirma que la falta de contacto o su carácter inadecuado entre padres-especialmente madres- e hijos era la razón principal de la aparición del trastorno en cuestión (Ramos, 2010).

Desde la década de 1970 en adelante se han desarrollado terapias basadas en la modificación de conducta. Si bien es cierto que el modelo conductista no propone una teoría de las causas de autismo, sin embargo se toma en cuenta porque instala una modalidad: la intervención terapéutica-educativa, que se mantiene hasta el presente y es considerada en el plano internacional como la más efectiva (Rutter, 1978).

Por otro lado, en la década de los 80' comenzaron a desarrollarse en Inglaterra explicaciones provenientes de corrientes cognitivas. Durante este tiempo se concibe al autismo como un déficit generalizado en las diferentes áreas del desarrollo cognitivo; sin embargo, a esta postura no faltaron autores que afirmaban que los trastornos cognitivos no pueden explicar los trastornos socio-afectivos (Rutter, 1978).

Se ha observado cómo a través de los años el autismo fue adoptando diferentes puntos de vista, críticas a las suposición de aparición, muchas de las posturas contribuyeron significativamente en el estudio riguroso del espectro autista, las cuales hasta el día de hoy mantienen vigencia, otras de ellas perdieron significancia y pasaron a ser aportaciones sin duda relevantes en su tiempo; sin embargo, no se puede dejar de lado los puntos de vista neurológico-genético. Éstas tienen importantes aportaciones al estudio del trastorno en cuestión, estudios específicos que han marcado hito en la historia del desarrollo autista (Riviere, 1997).

Según la neurobiología del autismo, se ha estudiado a partir de cinco fuentes de información: genética, radiológica, neurofisiológica, neuroquímica y neuropatológica. Éstas han mostrado anormalidades estructurales poco consistentes y sin utilidad diagnóstica en el momento.

Los estudios neuroquímicos evidencian alteraciones en los sistemas serotoninérgicos, gabaérgico, catecolaminérgico, colinérgico, entre otros. Estos estudios también dieron resultados sin especifidad o valor diagnóstico.

Por otro lado, desde el área histoanatómica, se han evidenciado alteraciones celulares del sistema límbico y el cerebelo, dando la posibilidad que exista una organización anormal de las sinapsis, que repercute en el funcionamiento de áreas cerebrales relacionadas con las deficiencias en la relación social, el lenguaje comunicativo, la preferencia por la permanencia y la resistencia al cambio. Asimismo, se ha manifestado que el cerebro de las personas con TEA tenían macrocefalia, 
es decir, el tamaño de la cabeza es más grande comparado al promedio correspondiente (cerebro anormal simétricamente) y reducción del tamaño de células del hipocampo, el subículo, porciones de la amígdala, los cuerpos mamilares y el núcleo septal medio. Seguidamente estudios de resonancia magnética han demostrado disminución del tamaño del vermis posterior y los lóbulos VI y VII del neocerebelo (Holguin, 2003).

De igual manera, los aspectos neuropsicológicos tratan de explicar los trastornos generalizados mencionando las siguientes características (Cabrera, 2007):

Déficit en las funciones ejecutivas: la afección de estas funciones puede ser la causa de las conductas repetitivas y de los intereses restringidos en los autistas.

Débil coherencia central: éste es un estilo de procesamiento cognitivo, que permite la observación holística, de esta manera, el autista tiende a hacer fijación en un solo estímulo de su mundo holístico, restringiéndolo así a la realidad.

Trastornos en la cognición social: El déficit en el reconocimiento de caras y expresiones faciales conlleva a los autistas a la incapacidad para discriminar emociones, así como para inferir en los deseos y las intenciones de los demás. En esta función es vital el trabajo de la amígdala, esta ceguera mental altera marcadamente la conducta social, lo que dificulta la "empatización".

\section{Definiciones sobre autismo}

Kanner, citado por Reviere (1997), menciona que el autismo surge con la presencia de tres trastornos relacionados entre sí. Son los trastornos cualitativos de la relación, alteraciones de la comunicación y lenguaje; asimismo, la falta de flexibilidad y el aspecto comportamental alterado.

Asimismo, el Departamento de Salud y Servicio Humano, citado en Meléndez (2009), afirma que el autismo se desarrolla durante los tres primeros años de vida, el cual resulta de un trastorno neurológico que afecta el funcionamiento del cerebro, impactando así a las áreas relacionadas con la interacción social y habilidades comunicativas.

Según el manual diagnóstico y estadístico de trastornos mentales DSM-IV (Ver cuadro 1), el autismo es un trastorno generalizado de desarrollo caracterizado por tres tipos de síntomas: alteración cualitativa de la interacción social, alteración cualitativa de la comunicación y patrones de comportamiento, intereses y actividades restringida, repetitiva y estereotipada.

\section{Cuadro 1}

\section{Criterios de Diagnóstico según el DSM-IV}

Criterios de Diagnóstico según el DSM-IV

A. Para darse un diagnóstico de autismo deben cumplirse 6 o más manifestaciones del conjunto de trastornos (1) de la relación,

(2) de la comunicación, (3) de la flexibilidad. Cumpliéndose como mínimo 2 elementos de (1), uno de (2) y uno de (3).

1. Trastorno cualitativo de la relación, expresado como mínimo en dos de las siguientes manifestaciones:

a. Trastorno importante en muchas conductas de relación no verbal, como la mirada de los ojos, la expresión facial, las posturas corporales y los gestos para regular la interacción social.

b. Incapacidad para desarrollar relación con iguales adecuadas al nivel evolutivo.

c. Ausencia de conductas espontáneas encaminadas a compartir placeres, intereses o logros con otras personas (ej: conductas de señalar o mostrar objetos de interés).

d. Falta de reciprocidad social y emocional.

2. Trastornos cualitativos de la comunicación, expresados como mínimo en una de las siguientes manifestaciones.

a. Retraso o ausencia completa de desarrollo de lenguaje oral (que no se intenta compensar con medio alternativos de comunicación. Como gestos, mímicas, etc).

b. En personas con habla adecuada, trastorno importante en la capacidad de iniciar o mantener comunicaciones.

c. Empleo estereotipado o repetitivo del lenguaje, o uso de un lenguaje idiosincrático.

d. Falta de juego de ficción espontáneo y variado, o de juego de imitación social adecuado al nivel evolutivo.

3. Patrones de conducta, intereses o actividad restrictiva, repetida y estereotipada, expresados como mínimo en una de las siguientes manifestaciones.

a. Preocupación excesiva por un foco de interés (o varios), restringido y estereotipado, anormal por su intensidad o contenido.

b. Adhesión aparentemente inflexible a rutinas o rituales específicos y no funcionales.

c. Estereotipias motoras repetitivas (ej: sacudidas de manos, retorcer los dedos, movimientos completos de todo el cuerpo, etc.).

d. Preocupación persistente por partes de objetos.

B. Antes de los 3 años, deben producirse retrasos o alteraciones de una de estas 3 áreas: (1) interacción social, (2) empleo comunicativo del lenguaje, (3) juego simbólico.

C. El trastorno no se explica mejor por un síndrome de Rett o trastorno desintegrativo de la niñez. 
Sin embargo, hay quienes consideran que el autismo tiene que ver con un trastorno emocional, producido por factores emocionales o afectivos inadecuados en la relación del niño con las figuras de crianza. Esos factores dan lugar a que la personalidad del niño no pueda constituirse correctamente $\mathrm{o}$, caso contrario, se trastorne (Riviére, 1997).

Finalmente Torres (citado en Meléndez 2009), menciona que el autismo se debe a una falta de relación social, carencia de intercambio afectivo, reiteración de rutinas compulsivas y una resistencia al cambio. Estos rasgos son los más notables dentro del espectro autista, ya que su interacción social es limitada.

\section{Dimensiones del espectro autista}

Se diferencian seis dimensiones en el espectro autista (Riviere, 1997):

\section{Trastornos de la relación social}

Aislamiento completo. No apego a personas específicas, a veces indiferenciación personas/cosas.

Impresión de incapacidad de relación, pero vínculo con algunos adultos. No con iguales.

Relaciones inducidas, externas, infrecuentes y unilaterales con iguales.

Alguna motivación a la relación con iguales, pero dificultad para establecerla por falta de empatía y de comprensión de sutilezas sociales.

\section{Trastornos de las funciones comunicativas}

Ausencia de comunicación, entendida como "relación intencionada con alguien acerca de algo".

Actividades de pedir mediante uso instrumental de las personas, pero sin signos.

Signos de pedir, solo hay comunicación para cambiar el mundo físico.

Empleo de conductas comunicativas de declarar, comentar, etc., que no solo buscan cambiar el mundo físico. Suele haber escasez de declaraciones internas y comunicación poco recíproca y empática.

\section{Trastorno del lenguaje}

Mutismo total o funcional (este último con emisiones verbales no comunicativas).

Lenguaje predominante ecolálico o compuesto de pa- labras sueltas.

Hay oraciones que implican creación formal espontánea, pero no llegan a configurar discurso o conversaciones.

Lenguaje discursivo. Capacidad de conversar con limitaciones. Alteraciones sutiles de las funciones comunicativas y la prosodia del lenguaje.

\section{Limitaciones de la imaginación}

Ausencia completa de juego simbólico de cualquier indicio de actividad imaginativa.

Juegos funcionales elementales inducidos desde fuera. Poco espontáneos, repetitivos.

Ficciones extrañas, generalmente poco imaginativas y con dificultades para diferenciar función/realidad.

Ficciones complejas, utilizadas como recursos para aislarse. Limitadas en contenidos.

\section{Trastornos de la flexibilidad mental y la conducta}

Estereotipias motoras simples (aleteo, balanceo).

Rituales simples, resistencia a cambios mínimos, tendencia a seguir los mismos itinerarios.

Rituales complejos, apego excesivo y extraño a ciertos objetos.

Contenidos limitados y obsesivos de pensamiento, intereses poco funcionales, no relacionados con el mundo social en sentido amplio y limitados en su gama.

\section{Trastornos del sentido de la actividad propia}

Predominio masivo de conductas sin propósito (correteos sin meta, ambulación sin sentido).

Actividades funcionales muy breves y dirigidas desde fuera, cuando no, se vuelve a (a).

Conductas autónomas y prolongadas de ciclo largo, cuyo sentido no se comprende bien.

Logros complejos (ej.: de ciclos escolares), pero no se integran en la imagen de un "yo proyectado en el futuro", motivos de logros superficiales, externos, poco flexibles.

A continuación en el siguiente cuadro se muestran las características en cuanto al lenguaje, comprensión, cognición y percepción pertenecientes al Trastorno del Espectro Autista. 


\section{Cuadro 2}

\section{Características específicas del TEA (Trastorno del Espectro Autista)}

\begin{tabular}{ll}
\hline \multicolumn{1}{c}{ Lenguaje y comprensión } & \multicolumn{1}{c}{ Falta de flexibilidad y adherencia a rutinas } \\
\hline Ausencia de lenguaje en algunos niños con autismo & Insistencia en la invariancia del ambiente \\
Uso extraño del lenguaje (ej: alteraciones sintácticas caprichosas) & Rígida adherencia a rutinas \\
$\begin{array}{l}\text { Ecolalia (tendencia a repetir de forma literal lo que oyen) } \\
\text { Tendencia a comprender el lenguaje de forma muy literal }\end{array}$ & $\begin{array}{l}\text { Un deseo ansioso y obsesivo de mantener la invariabilidad } \\
\text { Fascinación por ciertos estímulos }\end{array}$ \\
$\begin{array}{l}\text { Inversión de pronombres personales, ej: la confusión entre los } \\
\text { pronombres Yo y Tú }\end{array}$ & Deseos de conservar los objetos de una cierta manera (ej: mue- \\
Falta de atención al lenguaje & bles de la casa) \\
& Comportamiento repetitivo \\
Falta de relevancia de su lenguaje & Preocupación por sus movimientos de manos \\
Anomalías prosódicas & Aleteo de manos \\
Lenguaje limitado & Dar vueltas sobre sí mismo \\
Vocalizaciones sin lenguaje & Balanceo repetitivo \\
Retraso en el desarrollo del habla & Andar en puntillas \\
Lenguaje caracterizado por su carácter memorístico y repetitivo \\
La articulación y la fonética están conservadas & Girar objetos en forma continuada \\
Lenguaje hablado, pero sin carácter comunicativo & Alinear juguetes u objetos obsesivamente \\
\hline
\end{tabular}

\section{Cuadro 3}

$\begin{array}{cc}\text { Cognición y percepción } & \text { Conducta en las relaciones sociales, factores de personalidad y } \\ \text { de comunicación }\end{array}$

\footnotetext{
Un síntoma asociado de primer orden lo constituye la disca- Ausencia de juego de ficción

pacidad intelectual

CI medio alrededor de 50 (en población normal es de 100) Ausencia de mímica facial apropiada

Tendencia a representar las realidades de forma fragmentaria No mira a los ojos

(ej: solo se intenta una rueda de un coche - una parte)

Incapacidad de percibir totalidades coherentes

Puede haber respuestas extras a estímulos sensoriales

Respuestas paradójicas a los estímulos auditivos

Fuerte rechazo a ciertos sonidos

Fuerte rechazo a tocar ciertas texturas

No responde a las personas

Ausencia de coorientación visual (mirar hacia donde mira otro)

Trata a las otras personas como si fuera objetos inanimados

Cuando los coges, no ayudan (es como levantar un peso muerto)

Autosuficiente, comportándose como si la gente no estuviera presente

La tendencia de las personas con autismo a guiarse exclusiva- Más feliz cuando se lo deja solo
}

mente por impulsos internos, ignorando el entorno

Manifiestan ciertos rasgos de inteligencia, "islas de competen- Hiperactividad (infancia)

cia”, es decir las áreas donde el niño tiene una habilidad normal

o incluso extraordinaria (ej: dibujo, cálculo, fechas, música)

Hipoactividad (adolescencia)

Humor lábil

Hetereoagresividad, comportamiento agresivo hacia otros (menos frecuente que la autoagresividad)

Limitación de sus relaciones sociales

Ausencia de sonrisa social

Falta de respuesta a peligros reales

Compulsividad

Baja tolerancia a la frustración

Ausencia de interés por juguetes

Crisis de agitación (con o sin causa aparente)

Impulsividad

En los niños, rabietas

Rechazos a ser tocados

Comportamientos muy pasivo o demasiado activo y nervioso

Rechazo a comidas nuevas

Prefieren alimentos blandos 


\section{Habilidades sociales}

Actualmente en la Psicología, como en otras especialidades, se ha demostrado e identificado la importancia de las habilidades sociales o de las relaciones interpersonales en el éxito o fracaso de las personas en la sociedad. A lo largo de la vida el hombre va adquiriendo una serie de habilidades que le permite adaptarse a su medio, tanto físico como social.

De acuerdo a un modelo teórico de las habilidades sociales derivado de la Psicología Social, Fernández y Carboles (citado en Pérez, Saavedra, Salum y Silva 2005), definen habilidades sociales como la capacidad que el individuo posee para percibir, comprender, descifrar y, posteriormente, a responder a determinados estímulos sociales en general, en especial aquellos que vienen del comportamiento de los demás.

Teniendo en cuenta el modelo de aprendizaje social, Huidobro, Gutiérrez y Condemarín (citado en Pérez, et al 2005), definen habilidades sociales como conductas y comportamientos que permiten relacionarse e interactuar con el medio de manera adecuada.

Las habilidades sociales se expresan en sentimientos, actitudes y conductas de seguridad y confianza, independencia, por ende de autonomía; asimismo, se puede decir que las habilidades sociales son aprendidas y recíprocas. Goldtein (citado por García 2005), entiende por habilidades sociales al conjunto de habilidades y capacidades para el contacto interpersonal y la situación de problemas de índole interpersonal, así como socioemocional, desde actividades considerablemente básicas hasta actividades avanzadas y complejas.

Por otro lado Monjas y Gonzales (1998), menciona que las habilidades sociales son capacidades o destrezas que posee un sujeto para la ejecución competente de una tarea interpersonal.

De acuerdo con Caballo (citado por Pérez, et al 2005), manifiesta que la asertividad y habilidades sociales son sinónimos o equivalentes en definición y si ocupan el nombre de habilidades sociales es debido al uso global. De una forma sencilla, se podría decir que el término habilidades sociales es el conjunto de capacidades y destrezas de actuación aprendida que se desarrolla en un contexto interpersonal, donde dicho aprendizaje se da por imitación, modelamiento, reforzamiento positivo, observación o aprendizaje vicario.

\section{Dimensiones}

Según Valles y Valles (citado en Pérez, Saavedra, Salum y Silva 2005).

\section{Tabla1}

\section{Componentes de las habilidades sociales}

Componente conductual

No verbales

Paralingüísticos

Verbales

Componente cognitivo

Habilidad de percepción social (ambiente de comunicación)

Variables cognitivas de la persona

Componente afectivo

Manifestaciones psicológicas

Afectivo-emocionales
Miradas, sonrisas, gestos, expresión facial, contacto físico, orientación corporal

Latencia de respuestas

Voz (volumen, timbre, claridad, velocidad, tiempo de respuesta, fluidez,etc.)

El habla, hablar en público, la conversación, habilidades heterosociales

Formalidad, calidez, privacidad, familiaridad, restricción y distancia.

Objetivos y motivación, competencia cognitiva, constructos personales, expectativas personales, locus de control, valores subjetivos de los estímulos, autocontrol

Tasa cardiaca, presión sanguínea, relajación, respiración, respuesta electrodermales, respuesta electromiográfica

Expresión de emociones, control de la ansiedad, habilidades de relajación. 


\section{Investigaciones realizadas sobre las habilidades sociales en niños del espectro autista}

A lo largo de la historia y de los inicios del estudio del autismo, se vinieron realizando diversas investigaciones, a continuación se presentan algunas de ellas. Passerino y Santarosa (2007), realizaron una investigación en la Universidad Federal de Rio Grande do Sul Brasil, con el objetivo de analizar la interacción social en autistas en ambientes digitales de aprendizaje. Ésta se dividió en 3 etapas y contó con 4 individuos con autismo, en niveles diferenciados de leve a moderado, cuyas edades fluctuaban entre los 15 y 28 años. Donde leve era G1 y moderado G2. Los resultados obtenidos indicaron que el G1 tuvo mayor y mejor respuesta de interacción social y comprensión de la tarea a diferencia del G2, éste último presentaba alteraciones ecolálicas y movimientos estereotipados durante toda la sesión, lo cual impedía la comprensión de la tarea y la poca interacción entre compañeros de equipo.

Por otro lado, Meléndez (2009), en una investigación cualitativa de tipo documental tuvo el objetivo de desarrollar la socialización en los estudiantes con autismo a través de actividades físicas. La muestra fue constituía por 71 participantes del espectro autista, los resultados obtenidos de esta investigación indican que a través de las actividades físicas se promueve la socialización.

Merino, et al (2008), realizaron una investigación de tipo experimental, cuyo objetivo estuvo basada en el papel de las habilidades sociales y comunicativas en la inserción laboral de las personas con TEA, la población estuvo conformada de 2 a 3 individuos con TEA en 9 diferentes empresas cada individuo contaba con un entrenador. Los resultados obtenidos demostraron que las habilidades sociales más relevantes para el empresario son aquellas relacionadas con la correcta ejecución de la tarea; asimismo, los individuos con TEA dan gran importancia a su correcta ejecución de la tarea y se muestran motivados y perfeccionistas en el desempeño de sus funciones.

Asimismo, Pérez y Williams (2005), llevaron a cabo una investigación de tipo experimental cuyo objetivo fue la creación de un programa integral para la ense- ñanza de habilidades en niños con autismo. Su población se basó en 3 estudiantes con niveles diferenciados de autismo. La intervención tuvo lugar durante los meses de junio- agosto, donde los casos representados por niñas son: F- Caso 1 y E- Caso 2; por otro lado, los meses de julio-agosto tuvo lugar el único caso de hombre identificado como D- Caso 3. Los resultados obtenidos destaca a los 3 participantes como aptos a la expresión de habilidades sociales, D- Caso 3 respondió mejor a F- Caso 1 y E- Caso 2, habiendo desarrollado más las habilidades cognitivas y conductuales.

Por otro lado, Salvador, Fuente y Álvarez (2009), realizaron un estudio en habilidades sociales en directores de centros escolares. La muestra está compuesta por 99 directores de centros educativos públicos de primaria y secundaria, se enviaron de forma aleatoria los cuestionarios de habilidades sociales. Los resultados obtenidos demuestran que el $49 \%$ de los directores del nivel primario mantiene un nivel 51\% en habilidades sociales a través de comportamientos en los que la "autoexpresión”, “decir no”, “iniciar interacciones” y “expresar enfado" mostrando así niveles significativos.

Finalmente García (2005), realizó una investigación cuyo objetivo principal era establecer relación entre las habilidades sociales y el clima familiar con el rendimiento académico, la población de dicha investigación constó de 205 estudiantes de la Universidad Particular de San Martín de Porres. Los resultados obtenidos arrojaron una correlación positiva y significativa entre habilidades sociales y el clima social en la familia, encontrándose también que no existe estadísticamente una correlación significativa entre habilidades sociales y clima social en la familia con el rendimiento académico.

\section{CONCLUSIONES}

Se concluye el trabajo teniendo en cuenta que estas personas sufren de ciertas limitaciones de interacción social, funciones comunicativas, flexibilidad mental, alteraciones motoras, problemas conductuales, entre otros, y que con un entrenamiento adecuado y brindando las herramientas necesarias, éstos pueden desarrollar habilidades sociales tan bien como cualquiera. 


\section{REFERENCIAS BIBLIOGRÁFICAS}

1. Caballo, V. (2005). Manual de evaluación y entrenamiento de las habilidades sociales. Madrid: Siglo XXI Editores.

2. Cabrera, D. (2007). Generalidades sobre el autismo. Redalyc, 1, 208-220. Recuperado de: http:// redalyc.uaemex.mx/redalyc/src/inicio/ArtPdfRed. jsp?iCve $=80609916$

3. Flores, T., Toro, J., Masana, J., Treserra, J. Masana, E., Udina, C. (2002). Manual de diagnóstico y estadístico de los trastornos mentales. España: MASSON S.A.

4. García, R. (2005). Habilidades sociales, clima familiar y rendimiento académico en estudiantes universitarios. Redalyc, 011, 63-74.

5. Holguin, J. (2003). Neurofisiología del autismo. Revista Neurológica, (37)3, 259-266.

6. Meléndez, E. (2009). Desarrollo de la socialización a través de la adquisición de destrezas deportivas para los estudiantes con autismo del nivel elemental. Tesis presentada para la obtención del grado de maestría. Universidad Metropolitana, Lima.

7. Merino, M., Gutiérrez. M., Olivar, J., Ramírez, C., Hortigüela, V., Martínez, A. (2008). El papel de las habilidades sociales y comunicativas en la inserción laboral de las personas con TEA. ETAPI. 1(1), S/P.

8. Monjas, M. y Gonzales, B. (1998). Las habilidades sociales en el currículo Ministerio de Educación, cultura y deporte. España. Recuperado
de:http://213.0.8.18/portal/Educantabria/RECURSOS/Materiales/Biblinter/HABILIDADES.pdf.

9. Passerino, L. y Santarosa, L. (2007). Interacción social en el autismo en ambientes digitales de aprendizaje. Redalyc, 20(1), 54-64.

10. Pérez, L. y Williams, G. (2005). Programa integral para la enseñanza de habilidades en niños con autismo. Psicothema, 7(002), 233-244.

11. Pérez, T., Saavedra, H., Salum, L. y Silva, F. (2005). Significado de las Habilidades Sociales para diversos actores de instituciones educativas en la ciudad de Temuco. Tesis presentada para la obtención del grado de licenciado en educación. Universidad Católica de Temuco, Chile.

12. Ramos, M. (2010). ¿Qué es el autismo? La experiencia de padres inmersos en la incertidumbre, intersecciones en antropología. Redalyc, 11(1), 77-88 Recuperado de: http://redalyc. uaemex.mx/redalyc/src/inicio/ArtPdfRed. jsp?iCve=179516771006.

13. Riviere, A. (1997). Desarrollo normal y autismo. University Arizona, 1(2), S/P Recuperado de: http://uacoe.arizona.edu/moll/vygotsky/ARTICLES/Riviere_autismo.pdf.

14. Rutter, M. (1978). Trastornos de lenguaje y niños autistas. Nueva York: Plenum Press.

15. Salvador, M., Fuente, M. y Álvarez, J. (2009). Las habilidades sociales en directores de centros escolares. Redalyc, 2(3), 275-288. 